\title{
A ROBUST ADAPTIVE DEAD-TIME COMPENSATOR WITH APPLICATION TO A SOLAR COLLECTOR FIELD ${ }^{1}$
}

\author{
Julio E. Normey-Rico* Carlos Bordons** Manuel Berenguel ** \\ Eduardo F. Camacho**
}

\author{
* Dept. Automação e Sistemas. Univ. Fed. de Santa Catarina, Brazil. \\ ** Universidad de Sevilla. Escuela Superior de Ingenieros. Dept. de Ingeniería de \\ Sistemas y Automática. Camino de los Descubrimientos s/n, 41092, Sevilla, Spain. \\ E-mails: julio, bordons, beren, eduardo@cartuja.us.es
}

\begin{abstract}
This paper describes an easy-to-use PI controller with dead-time compensation that presents robust behaviour and can be applied to plants with variable dead-time. The formulation is based on an adaptive Smith predictor structure plus the addition of a filter acting on the error between the output and its prediction in order to improve robustness. The implementation of the control law is straightforward, and the filter needs no adjustment, since it is directly related to the plant dead-time. An application to an experimentally validated nonlinear model of a solar plant shows that this controller can improve the performance of classical PID controllers without the need of complex calculations. Copyright (C) 1998 IFAC
\end{abstract}

Keywords. Dead-time compensation, robust pi control, solar power plant

\section{INTRODUCTION}

Although PID controllers are the most frequently employed controllers in industrial practice, they show poor performance when the process exhibits long dead-times (Hägglund, 1996; Aström and Hägglund, 1995). In practice, where plant uncertainties are always present, the robust performance of the closed loop system is one of the most important control specifications. There are several techniques that can be used to design a controller with robust behaviour (Morari and Zafirou, 1989; Skogestad and Postlethwaite, 1996) but when the plant has long dead-time, predictive control can be the most efficient solution. In general, the solution given by predictive control is simpler to compute and to tune than the controller obtained using other techniques, like $\mathcal{H}_{\infty}$ or LQG/LTR.

Model-based predictive control is increasing its popularity, both in the academic world and in-

\footnotetext{
1 Work supported in part by CAPES-Brasil Contract BEXO448/95-6, CICYT-Spain TAP 95-370 and IBERDROLA (Ayudas a la Investigación Científica y al Desarrollo Tecnológico - Proyecto 96/036) within the project "Enhancement and Development of Industrial Applications of Solar Energy Technologies", supported by EEC Program "Human Capital and Mobility" and promoted by CIEMAT - Plataforma Solar de Almería, Spain.
}

dustry, although it is not completely accepted in industrial practice, mainly due to its relatively complex formulation. The main ideas of model predictive control can be used in a simpler controller, such as the predictive PI controller, where the most common dead-time compensating controller, the Smith predictor (SP, Smith, 1957) is combined with the simple and extensively used PI. This constitutes a simple control structure, in which the tuning procedure can be simplified if the number of adjustable parameters is reduced, as proposed by Hägglund (1996).

Normey-Rico et al. (1997), analyze the robustness of the predictive PI controller proposed by Hägglund (1996) for the case of stable plants, and propose a simple way to enhance it, improving the behaviour when the process does not fit the nominal model and maintaining the nominal performance. Besides, the formulation does not include extra complexity, since the addition consists of a low-order filter that is internally tuned and can be found in any commercial controller. The controller has only three tuning parameters that can be tuned using a classical step identification test. The behaviour of this filtered predictive PI controller (FPPI) was tested in simulation and in real-time applications.

In practice, there are several industrial processes 
where the dead-time is caused by the time taken for some material to be transferred from the position of the supply to the position of the sensor. In these cases the dead-time varies inversely proportional to the velocity of transportation of the material. Thus, the measurement of this velocity can be used to estimate the dead-time of the plant.

This paper presents a successful application of an adaptive robust predictive PI controller to a experimentally tested nonlinear model of the ACUREX distributed collector field of the Plataforma Solar de Almería (Southern Spain) used for comparison purposes, showing its simplicity and performance.

The paper is organized as follows: first a short review of the FPPI controller is given in Section 2 . Section 3 deals with the consideration of deadtime variations and gives a procedure to estimate the dead-time. A modified nonlinear model of the ACUREX solar arrays based on first principles is briefly explained in section 4 , while in section 5 the results of the tests carried out using the proven nonlinear model are presented. The paper ends with some concluding remarks.

\section{ROBUST PREDICTIVE CONTROLLERS}

Dead-times appear in many industrial processes, usually associated with mass or energy transport, or due to the accumulation of a great number of low-order systems. Dead-times produce an increase in the system phase, therefore decreasing the phase and gain margins. When using classical methods to control processes where the effect of dead-time is dominant, the controller must be detuned to achieve stability, providing a slow behaviour. In these cases it is convenient to use a dead-time compensating method. The SP is perhaps the best known and most widely used algorithm for dead-time compensation. With this structure, if there are no modelling errors or disturbances, the error between the current process output and the model output will be null and the controller could be tuned as if the plant had no dead-time. The most commonly used plant model in the SP is a first-order system with an effective dead-time, since most processes in industry can be described in this way. These systems can be described by the following transfer function, consisting of a static gain $K_{p}$, a time constant $T$ and a effective dead-time $L: P(s)=K_{p} e^{-L s} /(1+T s)$.

With the addition of the PI, the resulting controller has five tuning parameters: the three plant coefficients $K_{p}, T$ and $L$, and the two PI parameters $K$ and $T_{i}$. This can constitute an increase in operational complexity compared with a PID controller, so Hägglund (1996) proposes a predictive PI controller (PPI) with only three adjustable parameters, choosing the controller gain equal to the

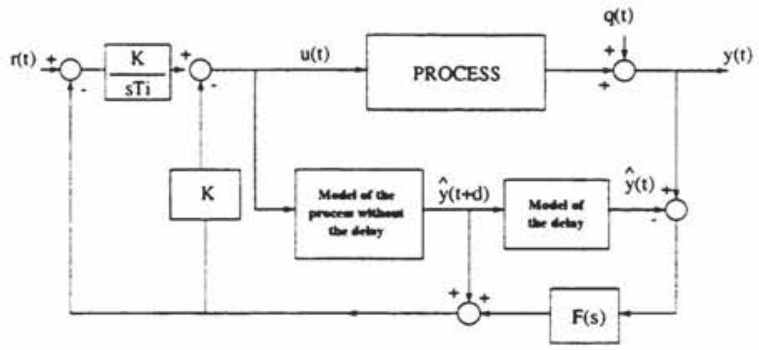

Fig. 1. Structure of the filtered PPI

inverse of the process gain and the integral time equal to the process time constant. This results in a simple controller that has the same advantages as the PID controllers, since it can be easily tuned manually, and also is suitable for the control of processes with long dead-times. However, this formulation does not take account of the robustness of the closed-loop system and presents stability problems when uncertainties exist in the plant parameters, mainly in the dead-time.

A robustness analysis of the PPI can be found in Normey-Rico et al. (1997) where the authors have shown that the PPI controller has undesirable closed-loop behaviour when dead-time uncertainties (high-frequency modelling errors) are considered. As these type of uncertainties are very common in the process industry, some modifications to the PPI controller were introduced to improve robustness: a filter $F(s)$ with unitary static gain $(F(0)=1)$ was introduced, as shown in Fig. 1, maintaining the structure of the PI controller proposed by Hägglund (1996). The filter $F$ can be used to improve the robustness of the system at the desired frequency region. If it is desired to maintain the simple structure of the PPI in order to use the filtered PPI (FPPI) controller in industrial applications, then the filter $F$ will be defined as a first-order filter with only one parameter (the time constant $T_{f}$ ) related to $L$. The relation between $T_{f}$ and $L$ was computed in NormeyRico et al. (1997) as $T_{f}=L / 2$. This choice of $T_{f}$ gives good results for error in the dead-time up to $30 \%$. However, if the changes in the deadtime are greater than $50 \%$ of the nominal value and robust closed-loop performance specifications are defined, the FPPI will not give a satisfactory solution. As in most of the industrial cases high varying dead-times are associated to the transport of mass or flow and so an adaptive scheme can be used to improve the performance of the FPPI as shown in the next section.

\section{THE ADAPTIVE FPPI}

Varying dead-times are present in several industrial processes, such as heat exchangers. A special case is a solar collector which uses a parabolic surface to concentrate a direct normal beam onto a receiver tube which is located at the focal point 
of the parabola. The heat transfer fluid is pumped through the receiver tube and picks up the heat transferred through the receiver tube walls. The schematic diagram of this special kind of heat exchanger is shown in Fig. 2, where the dead-time is a function of the flow $(f)$, and the length $(l)$ and section $(S)$ of the tube.

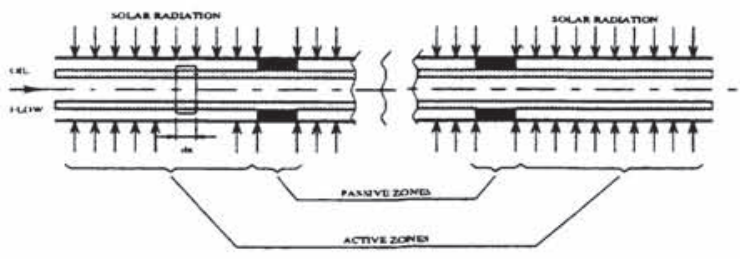

Fig. 2. Schematic diagram of a solar collector

This type of processes can be modelled by a first order transfer function plus a dead-time $L$. Part of this dead-time is used to model the effect of high order dynamics and the other part correspond to the real transport of fluid. Thus the estimated dead-time $L$ can be computed as $L=L_{0}+g(f)$. It is assumed that $L_{0}$ has only small variations around its nominal value (less than $30 \%$ ) and the FPPI can cope with the effects of these model uncertainties. The relation between the flow and the dead-time, $g(f)$, will be analyzed in the following.

In steady state conditions, the residence time of the fluid within the pipe is given by $t r=l / v, v$ being the constant fluid velocity $(v=f / S)$. Nevertheless, the flow values change during the daily operation, so that the value of the residence time has to be obtained by using it as an upper limit in the integration of the fluid velocity (equation (1)).

As the flow values are changed each sampling time, an approximation can be made to estimate the transport delay of the system as an integer multiple $n$ of the sampling time. Each sampling time $k$, a new value of the required flow $f(k)$ is calculated by the controller. The distance $d x_{k}$ that can be covered by the fluid during one sample $\left(T_{s}\right)$ with corresponding flow $f(k)$ is given by: $d x_{k}=$ $T_{s} f(k) / S$. Using a discrete-time approximation of equation which accounts for different values of the flow during different sampling intervals, the value of $n$ can be computed each sampling as follows:

$$
l=\int_{0}^{t_{r}} v(t) d t \rightarrow \frac{T_{s}}{S} \sum_{i=1}^{i=n} f(i)=l,
$$

where values of $f(i)$ are known from the previous measured ones $(f(1)$ is the actual flow, $f(2)$ was the flow demanded the previous sampling time and consequently, $f(n)$ was the flow demanded $n$ previous sampling times so that $n T_{s} \approx t_{r}$ ). Thus, the dead-time of the process can be estimated by the following relation: $L=L_{0}+n T_{s}$.

Notice that the control scheme is quite easy to use as: (i) the digital implementation of the previous relationship is very simple and does not need any tuning parameter; (ii) the complete tuning procedure for the controller is the same as in the FPPI and only the values of $l$ and $S$ must be given for the adaptive part of the control law; (iii) in general, the estimated value of $L$ will be different from the real dead-time because of the error in the measurement of $l$ and $S$, but as will be shown in the section 5 , the filter can cope with these modelling errors.

\section{COLLECTOR MODEL INCLUDING VARYING TRANSPORT DELAY}

As has been mentioned, the FPPI has been tested using a nonlinear model of the ACUREX field of the Plataforma Solar de Almería (Spain). The objective of the control system in a distributed collector field is to maintain the outlet oil temperature at a desired level in spite of disturbances such as changes in the solar irradiance level (caused by the daily variation and passing clouds), mirror reflectivity or inlet oil temperature. Since solar radiation cannot be adjusted, this can only be achieved by adjusting the flow of oil, and the daily solar power cycle characteristic is such that the oil flow has to change substantially during operation. This leads to significant variations in the dynamic characteristics of the field, such as the response rate and the time delay, which cause difficulties in obtaining adequate performance over the operating range with a fixed parameter controller. A schematic diagram of the field is given in Fig. 3.

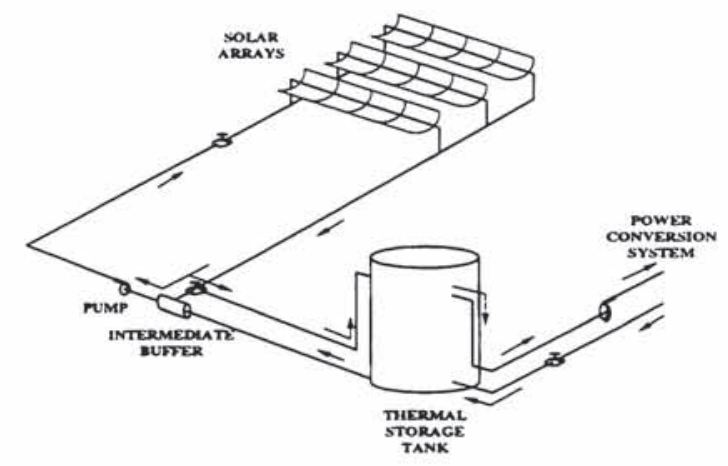

Fig. 3. Schematic diagram of the ACUREX field

From the control viewpoint, in order to assure a safe operation, the controlled variable in this kind of plants is usually the outlet temperature of the loop with the highest value at each sampling time. Nevertheless, situations may exist in which the hot oil should be used for directly feeding the heat exchanger of the electricity generation system or a desalination plant. In these situations it is preferable to control the temperature 
of the oil entering the top of the thermal storage tank. In this way, the considerable length of the tube joining the output of the collector field with the top of the tank introduces a large variable delay within the control loop which depends on the value of the flow. As pointed out by Aström and Hägglund (1995), systems with dominant time delays are candidates for more sophisticated control than a simple PID. As it is commented in the next section, systems with time delays can sometimes be (poorly) controlled by using detuned PID controllers (there seems to be general agreement that derivative action does not help much for processes with dominant time delays (Aström and Hägglund, 1995)), but the performance can be improved by including some kind of dead-time compensation, as it is done in this paper.

The plant can be described by a set of nonlinear distributed parameter equations describing energy and mass balance. A nonlinear distributed parameter model was developed so that the control scheme could be tested by simulation (Camacho et al., 1988, Berenguel et al., 1993). Under some conditions the temperature in the field can be given by the following equations:

$$
\begin{aligned}
\rho_{m} C_{m} A_{m} \frac{\partial T_{m}}{\partial t}= & I n_{o} D-H_{1} G\left(T_{m}-T_{a}\right)- \\
& -L H_{t}\left(T_{m}-T_{f}\right) \\
L H_{t}\left(T_{m}-T_{f}\right)= & \rho_{f} C_{f} A_{f} \frac{\partial T_{f}}{\partial t}+\rho_{f} C_{f} \dot{q} \frac{\partial T_{f}}{\partial x}
\end{aligned}
$$

where the subindex $m$ refers to the metal and $f$ to the fluid. The rest of the parameters are as follows: $\rho$ : density, $C$ : field capacity, $A$ : transversal area, $T$ : outlet temperature, $I$ : solar radiation, $n_{o}$ : optical efficiency, $H_{1}$ : overall thermal loss coefficient, $D$ : mirrors width, $H_{t}$ : coefficient of metal fluid transmission, $G$ : exterior diameter of the pipe line, $L$ : inner diameter of the pipe line, $\dot{q}$ : oil flow. These equations are only applicable to the active zones of the field, that is, those parts of the pipe line where solar radiation is collected. Parts of the field, passive zones, exist where it is not possible to collect solar energy due to geometrical conditions as is the case of the joints between the modules. These zones constitute a considerable part of the field and they are characterized by having nil irradiance and different loss constants. The above equations were used to simulate the system in a computer dividing one of the loops into one hundred pieces and using a model of concentrated parameters for each piece. The model was contrasted to the real data obtained from the field (Camacho $e t$ al., 1988, Berenguel et al., 1993).

In order to use the previous model to account for longer passive tube lengths for comparing different dead-time compensation control schema, some modifications had to be performed. The nonlinear

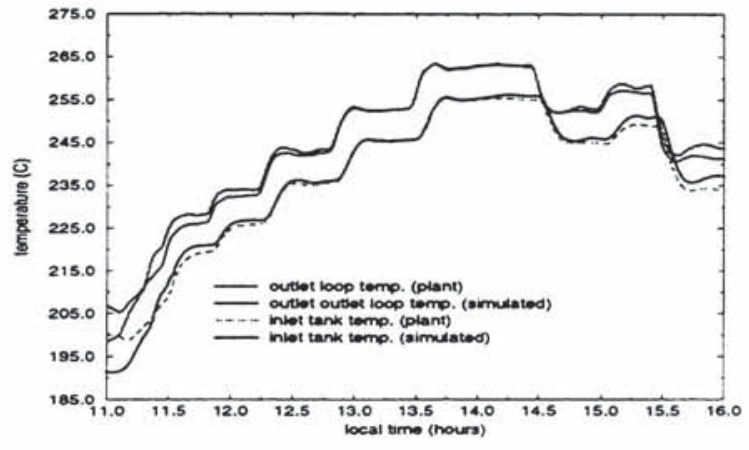

Fig. 4. Validation of the nonlinear model

discrete model of the field has provided very appreciate results when controlling the output temperature of the collector loops, as in this case the delay is small compared to the characteristic time constant of the plant. In this sense, a fixed integration step was used in the discrete model formulation based on a Euler transformation. If a variable integration step is to be used, this type of transformation suffer from numerical instability for integration step lengths needed from transport delay considerations when controlling the temperature entering the top of the storage tank. Alternative models based on other type of transformations can be developed (Meaburn and Hughes, 1993) and even including some kind of storage hold facility into the model. One approximated way to cope with the variable time-delay is by using the equations (1) developed in the previous section. In this way transport delay characteristics can be included in the nonlinear simulation model. As shown in (Rato et al., 1997), the dynamic characteristics of the tube joining the output of the loops with the top of the storage tank are given by a gain less than one, a time constant (of about 2 or 3 minutes) and a variable delay. This approximation has been adopted in order to modify the basic formulation of the nonlinear model to account for dynamic characteristics introduced by the tube. The modified model has been validated with data obtained at the plant in closed-loop operation (Rato et al., 1997). As an example, Fig. 4 shows the results obtained in closed-loop operation at the ACUREX field. Both the outlet temperature of the collector field and the inlet temperature at the top of the storage tank are shown and compared with those obtained from the nonlinear modified model. As can be seen, results are quite approximated (mostly around solar midday) and so the model has been used as an appropriate test-bed for dead-time compensation control schema.

\section{COMPARATIVE RESULTS}

To illustrate the performance of the FPPI controller some simulation results are given in this section. The solar plant has been controlled with 
a large number of control schema, some of which are resumed in (Camacho et al., 1997), most of all accounting for the control of the outlet oil temperature of the collector arrays. One well-tuned PID controller has shown to provide quite good performance in a wide range of operating conditions, although the behaviour deteriorates when operating at low flow conditions. Other conservative PI controller has been largely used as a backup controller and for comparison purposes which provides acceptable performance in the whole range of operating conditions. All these controllers incorporate a series feedforward term based on steady-state relationships aimed at compensating measurable disturbances acting on the plant, mainly solar radiation changes due to the solar daily cycle and passing clouds and inlet oil temperature changes due to non-uniformities at the bottom of the thermal storage tank.

When the controlled variable is the temperature of the oil entering the top of the storage tank, classical PID control schema performance deteriorates, as the dead-time dynamics become dominant. As has been pointed out by Aström and Hägglund (1995), control of systems with a dominant time delay are notoriously difficult. One way to cope with this problem is to detune PID controllers to obtain a stable operation in the whole range of operating conditions and with a bounded set of possible values of the varying delay. The problem in this case is that safe operation is obtained but as a consequence of a sluggish or slow response. Most of all, if some transient specifications have to be fulfilled around a determined operating point, undesirable results can be achieved around other extreme circumstances. The well-tuned PID controller (Camacho et al., 1997) used for controlling the outlet temperature of the field has been detuned to achieve adequate performance when controlling the temperature of the oil entering the top of the storage tank. Two sets of parameters have been obtained. In a first step in the design of the PID controller, only the integral time of the PID controller has been augmented and made equal to the dominant time constant of the system obtained from the identification of a step test around medium flow conditions. As a consequence, an acceptable response has been obtained when operating around these conditions but poor performance is obtained when operating at low flow conditions (at the starting and final phase of the operation, when the solar radiation values are low and suffer from great changes). This fact can be seen in Fig. 5(a) in which the outlet temperature with the detuned PID controller is shown. If a safe operation is to be achieved with a PID controller, more conservative changes in the PID controller parameters have to be performed (Fig. 5(b)), producing slow responses in many parts of the operation and making the tuning process quite time consuming.

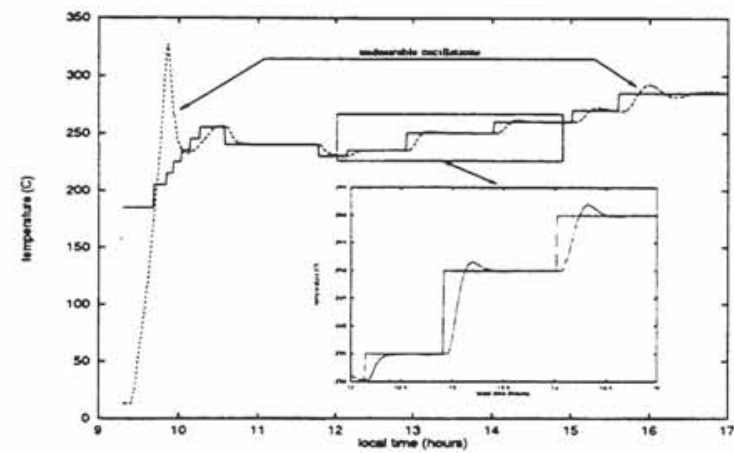

(a) first detuned PID

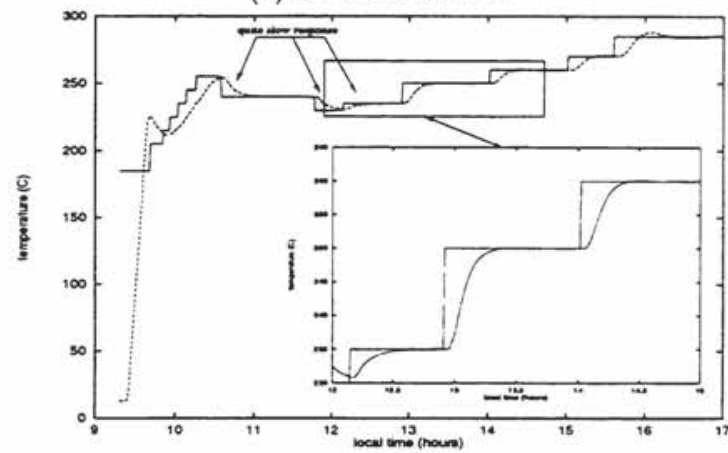

(b) second detuned PID

Fig. 5. Results with a detuned PID controller

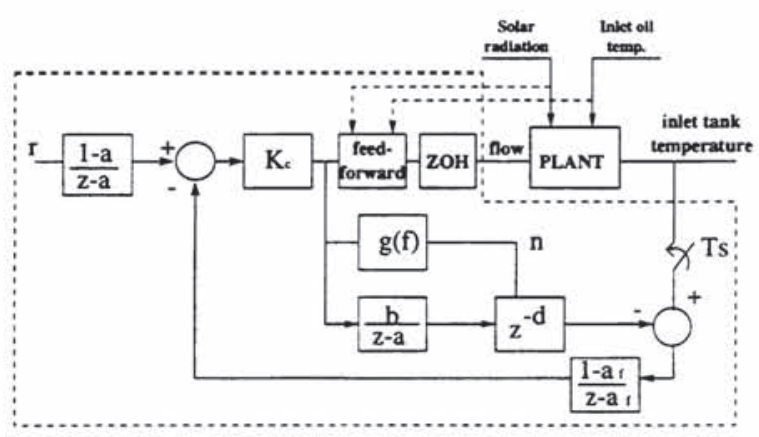

Fig. 6. Control scheme for the solar arrays

The performance of classical PID controllers can be improved by including dead-time compensation. The complete control structure proposed for the adaptive FPPI is shown in Fig. 6 for a digital implementation, where $a=e^{-T_{s} / T}, b=K(1-a)$; $a_{f}=e^{-T_{s} / T_{f}}$ and $d$ is the discrete-time representation of the dead-time. The identification of step tests has been used to obtain different models of the plant without the varying dead-time for different operating points. In the application shown in this paper the model for the predictor without the varying dead-time has been chosen as $P_{n}(s)=$ $\frac{1.2 e^{-78 s}}{1+320 s}$, and so the control parameters were: $K=$ $0.833, T i=320$ seconds and $L_{0}=78$ seconds. The sampling time was chosen $T_{s}=39$ seconds and the measurement of the equivalent length and section of the tube (which at the solar plant has different sections at different parts of the tube) 


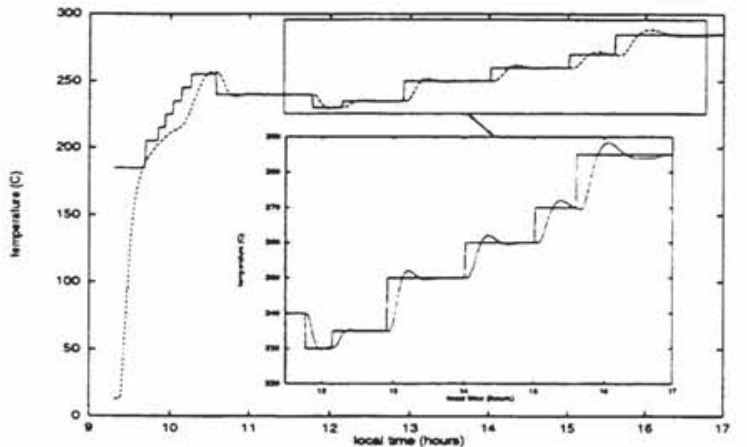

(a) outlet oil temperature $\left({ }^{\circ} \mathrm{C}\right)$

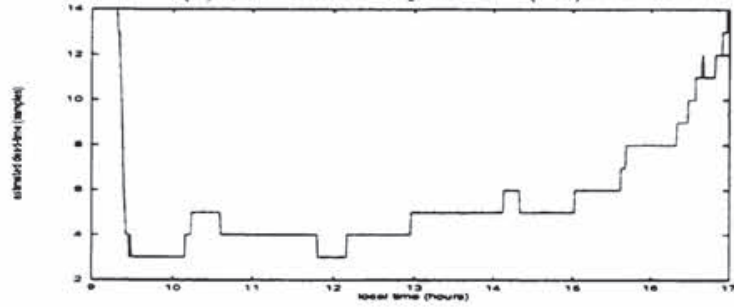

(b) estimated dead-time (sampling times)

Fig. 7. Results with the nominal FPPI

gives the following values: $l=200 \mathrm{~m}$ and $S=$ $5.3092910^{-4} \mathrm{~m}^{2}$. The time constant of the filter $\left(T_{f}\right)$ was chosen according to the value of the estimated delay using the procedure explained in section 3 . With these parameters the performance of the FPPI controller has been analyzed using the data file with which the simulations shown in Fig. 5 have also been performed. In Fig. 7 the results obtained with the basic design of the FPPI are presented. As can be seen, the controlled system accounts for the estimated varying dead-time (which varies according to oil flow) and assures an acceptable good operation in the whole range of working conditions. It must be noted that the tuning of the adaptive FPPI is simpler than the procedure used to obtain the detuned PID and gives better performance and robustness.

To analyze the effect in the performance when dead-time estimation errors occur, a non-adaptive FPPI controller was implemented by using two extreme fixed values of the dead-time: one (12 sampling times) near the maximum possible value and other (4 sampling times) near the minimum one, obtaining the results shown in Fig. 8. As can be seen, the performance deteriorates but the control scheme can cope with the operation covering a wide range of operating conditions without leading the system to instability, proving the robustness inherent in the design of the controller.

\section{CONCLUSIONS}

A simple and effective adaptive robust compensating PI controller has been presented. The controller has only three adjustable parameters that can be tuned manually or using some information about the plant with quite low commission-

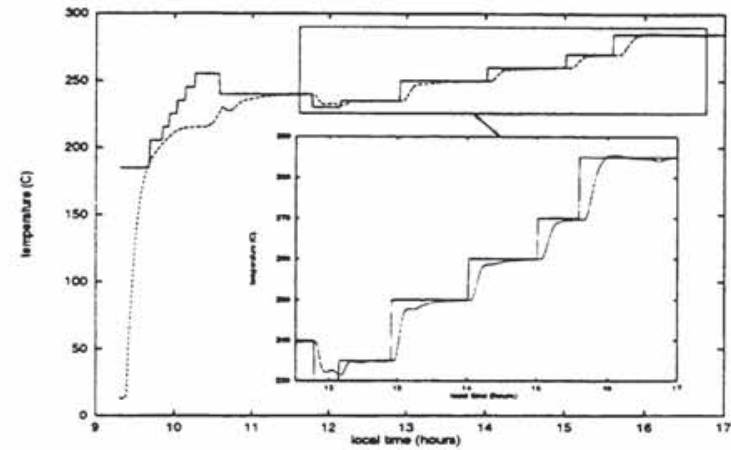

(a) maximum dead-time

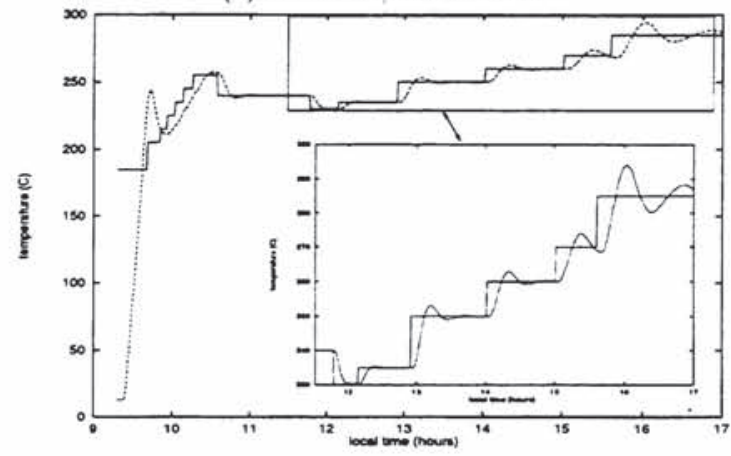

(b) minimum dead-time

Fig. 8. Results with the fixed dead-time FPPI

ing time. The proposed controller is suitable for processes with long dead-times and parameter uncertainties, commonly found in the process industry. The results obtained from the application to a simulated solar plant with arrays of parabolic trough collectors confirm the good qualities of the proposed controller.

\section{References}

Aström, K.J. and T.Hägglund (1995). PID Controllers: Theory, Design and Tuning. Inst. Society America.

Berenguel, M., E.F. Camacho and F.R. Rubio (1993). Simulation Software Package of the Acurex Field. E.S.I. of Seville, http://urw.esi.us.es/-beren.

Camacho, E.F., F.R. Rubio and J.A. Gutierrez (1988). Modelling and Simulation of a Solar Power Plant with a Distributed Collector System". In Int. IFAC Symp. on Power Systems Modelling and Control Applications. Brussels, pp. 11.3.1-11.3.5.

Camacho, E.F., M. Berenguel and F.R. Rubio (1997). Advanced Control of Solar Plants. Springer London.

Hägglund, T. (1996). An industrial Dead Time Compensating PI Controller. Cont. Eng. Practice 4, 749-756.

Meaburn, A. and F.M. Hughes (1993), Resonance Characteristics of distributed solar collector fields, Solar Energy, vol. 51, n. 3, pp. 215-221.

Morari, M. and E. Zafiriou (1989). Robust Process Control. Prentice-Hall, Englewood Cliffs.

Normey-Rico, J.E., C. Bordons and E.F. Camacho (1997) Improving the robustness of dead-time compensating PI controllers, Control Eng. Practice, 6, 801-810.

Rato, L., R.N. Silva, J.M. Lemos and F.J. Coito (1997). Multirate MUSMAR cascade control of a distributed collector solar field. ECC'g7, Brussels.

Skogestad, S. and I. Postlethwaite (1996). Multivariable Feedback Control. John Wiley and sons.

Smith, O.J.M. (1957). Closed control of loops with deadtime. Chem. Eng. Progress 53, 217-219. 